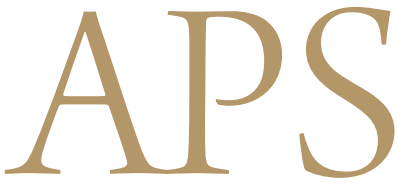

Archives of Plastic Surgery

\title{
Glomus Tumors: Symptom Variations and Magnetic Resonance Imaging for Diagnosis
}

\author{
Ki Weon Ham, In Sik Yun, Kwan Chul Tark \\ Department of Plastic and Reconstructive Surgery, Yonsei University College of Medicine, Seoul, Korea
}

Background The typical clinical symptoms of glomus tumors are pain, tenderness, and sensitivity to temperature change, and the presence of these clinical findings is helpful in diagnosis. However, the tumors often pose diagnostic difficulty because of variations in presentation and the nonspecific symptoms of glomus tumors. To the best of our knowledge, few studies have reported on the usefulness of magnetic resonance imaging (MRI) in diagnosing glomus tumors in patients with unspecific symptoms.

Methods The inclusion criteria of this study were: having undergone surgery for subungual glomus tumor of the hand, histopathologic confirmation of glomus tumor, and having undergone preoperative MRI. Twenty-one patients were enrolled. The characteristics of the tumors and the presenting symptoms including pain, tenderness, and sensitivity to temperature change were retrospectively reviewed.

Results Five out of 21 patients (23\%) did not show the typical glomus tumor symptom triad because they did not complain of pain provoked by coldness. Nevertheless, preoperative MRI showed well-defined small soft-tissue lesions on T1- and T2-weighted images, which are typical findings of glomus tumors. The tumors were completely resected and confirmed as glomus tumor histopathologically.

Conclusions Early occult lesions of glomus tumor in the hand may not be revealed by physical examination because of their barely detectable symptoms. Moreover, subungual lesions may be particularly difficult to evaluate on physical examination. Our cases showed that MRI offers excellent diagnostic information in clinically undiagnosed or misdiagnosed patients. Preoperative MRI can accurately define the character and extent of glomus tumor, even though it is impalpable and invisible.

Keywords Glomus tumor / Neoplasm / Hand

Correspondence: Kwan Chul Tark Department of Plastic and Reconstructive Surgery, Yonsei University College of Medicine, 50 Yonsei-ro, Seodaemun-gu, Seoul 135-710, Korea

Tel: +82-2-2228-2210

Fax: +82-2-393-6947

E-mail: kctark@yuhs.ac

Received: 15 Mar 2013 • Revised: 19 Apr 2013 • Accepted: 25 Apr 2013

plSSN: 2234-6163・elSSN: 2234-6171・ http://dx.doi.org/10.5999/aps.2013.40.4.392・Arch Plast Surg 2013;40:392-396

This article was presented at the Congress of the Korean Society of Plastic and Reconstructive Surgeons on November 12, 2011 in Seoul, Korea.

No potential conflict of interest relevant to this article was reported.

\section{INTRODUCTION}

A glomus tumor is a rare, benign, small vascular tumor that arises from specialized cells normally found within the glomus (Latin for "ball" or spherical mass) body in the reticular dermis.
The most common site for glomus tumor development is the distal phalanx, particularly beneath the nails; however, this tumor can be found anywhere on the body [1]. A glomus body is composed of an afferent arteriole, anastomotic vessel (SucquetHoyer canal), primary collecting vein, intraglomerular reticu-

Copyright ( $\odot 2013$ The Korean Society of Plastic and Reconstructive Surgeons

This is an Open Access article distributed under the terms of the Creative Commons Attribution Non-Commercial License (http://creativecommons.org/

licenses/by-nc/3.0/) which permits unrestricted non-commercial use, distribution, and reproduction in any medium, provided the original work is properly cited.

www.e-aps.org 
lum, and capsular portion [2]. Tumors can develop as a result of hyperplasia in any of these parts. The normal glomus body is a contractile neuromyoarterial receptor that controls blood pressure and temperature by regulating peripheral blood flow. Present in the stratum reticularis of the dermis throughout the body, glomus bodies are highly concentrated in the tips of the digits, especially under the nails $[3,4]$.

The typical clinical symptoms of glomus tumor of the finger are localized tenderness, severe paroxysmal pain, and cold sensitivity. Despite this knowledge, clinicians often have difficulty diagnosing this condition because of variations in symptom presentation and nonspecific symptoms of glomus tumors. It is not uncommon for patients to remain undiagnosed or misdiagnosed for many years. In such cases, an imaging modality, such as magnetic resonance (MR) imaging, can provide clues. To the best of our knowledge, few publications have reported on the usefulness of MR imaging in diagnosing glomus tumors in patients exhibiting nonspecific symptoms. In this study, we present 21 cases of glomus tumor of the hand for which MR imaging played a critical role in forming an accurate diagnosis.

\section{METHODS}

The 21 patients included in this study, seventeen women and four men, underwent surgery for subungual glomus tumor of the hand between January 1995 and December 2012. The mean age of the patients at the time of surgery was 48.4 years (ranging from 36 to 78 years), and the mean duration of symptom manifestation was 18 months (ranging 4 to 52 months). The surgeries were performed under general anesthesia and with a tourniquet technique. We used standard direct transungual excision, in which the nail plate was removed using a Freer elevator and incised longitudinally with a No. 15 blade directly over the area of the tumor. The tumor was excised sharply with a No. 15 blade, and the distal phalanx bone was scraped to ensure complete removal. The nail was saved; after removing the tumor, the nail was inserted back into its original location.

All 21 patients suspected of having glomus tumor of the fingertip were examined with MR imaging before surgical exploration. Both T1- and T2-weighted images were acquired for all patients and the images were also examined after IV administration of contrast medium. All of the patients exhibited spontaneous paroxysmal pain and complained of tenderness; upon touching; however, only 16 patients experienced worse pain upon exposure to cold temperatures. Therefore, 5 (23\%) of 21 patients did not exhibit the typical symptoms of glomus tumors. The final diagnosis was established in all of the patients with a pathologic evaluation of samples resected by expert histopathologists.

\section{RESULTS}

Comparing the preoperative evaluation results and postoperative histopathology of the 21 patients including the group with ambiguous symptoms, preoperative MR imaging was shown to be important in that it complemented the insufficient clues gained only through physical examination.

In fact, among the 21 patients only 6 of the patients (28.6\%) came to our institution by themselves or were diagnosed with a glomus tumor at other institutions, while 15 patients (71.4\%) were misdiagnosed upon physical examination only according to symptoms and signs.

The preoperative clinical diagnosis of glomus tumor was determined in the 21 patients based on histopathology, and MR imaging confirmed each of these diagnoses. Positive MR imaging showed localization of a hypo-intense (dark) lesion that was well-marginated and oval-shaped on T1-weighted images. On T2-weighted images, these lesions appeared brighter (higher signal intensity) and hyper-intense with a hypo-intense rim. Nevertheless, we were able to obtain T1-weighted images after enhancement through IV administration of contrast medium.

MR imaging was a decisive clue in settling on the surgical approach in patients without a confirmed diagnosis using the aforementioned symptoms, and also, was also an important modality in deciding the target of surgical treatment.

The MR imaging results correlated with tumor lesions identified histopathologically. In this study, MR imaging had a 100\% positive predictive value for finding small mass lesions as a preoperative test. All of the patients were examined again at least 6 months later. No recurrence or postoperative complications due to wound infection or nail bed deformity were reported. Moreover, all of the patients were relieved of painful symptoms immediately after surgery.

\section{Case reports}

Case 1

This 45-year-old male patient experienced intermittent pain and tenderness for 2 years, without pain provoked by coldness (Fig. 1). He presented with definite localized tenderness, but resting pain at the fingertip area was ambiguous, intermittent and slightly progressed. Upon physical examination, we could not find any nail plate deformity or color change in the subungual area. Despite nonspecific symptoms on the fingertip area, and taking into account the long-term pain of this patient, we conducted a non-invasive MR imaging study prior to surgical exploration. The T1-weighted MR images showed a well-marginated, round, hypo-intense lesion located in the subungual region of the fourth finger. The T2-weighted images revealed a hyper- 
intense lesion with a hypo-intense rim (Fig. 2). The mass on the fingertip was approximately $3 \mathrm{~mm}$ in diameter. MR imaging, strongly suggested that the lesion was a glomus tumor. Using MR imaging, we were able to plan the surgical approach to the lesion. The mass was excised completely through surgery and verified histopathologically to be a glomus tumor (Fig. 3). The localized pain and point tenderness disappeared immediately after surgical excision. The patient did not experience any recurrence or further complications.

\section{Fig. 1. Preoperative photo of case 1}

The 45-year-old male patient had experienced localized pain and tenderness for 2 years, without pain provoked by coldness.

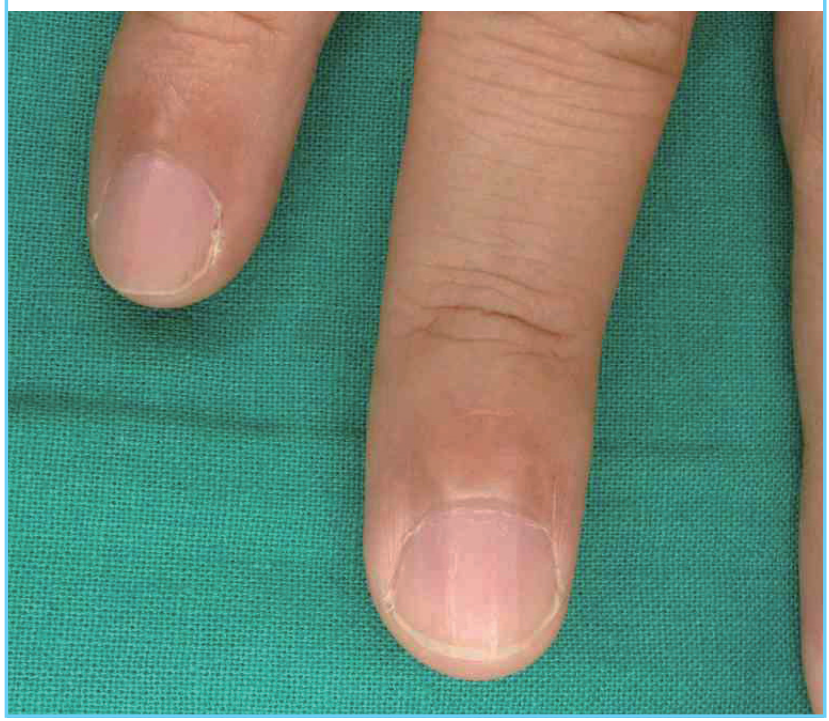

\section{Fig. 2. Preoperative MRI of case 1}

Preoperative magnetic resonance imaging (MRI) of case 1 (T2weighted), which revealed a hyper-intense lesion (white arrow) with a hypo-intense rim.

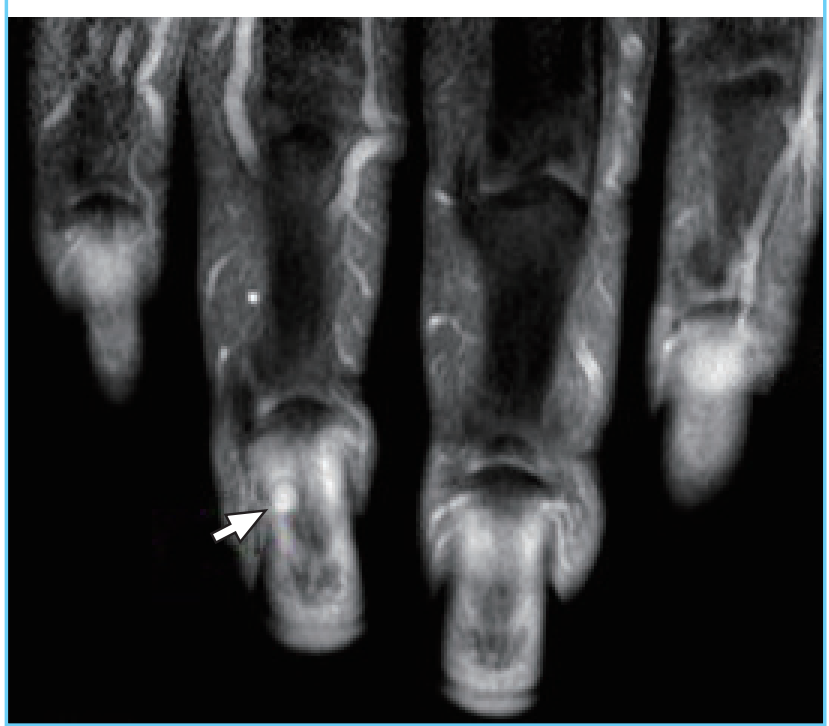

\section{Case 2}

A 78-year-old female patient suffered point pain, tenderness aggravated by cold irritation, and nail irregularity in the thumb (Fig. 4). As described above, this patient exhibited the triad of symptoms of glomus tumor. However, she additionally presented with severe paroxysmal pain and radiating pain to the wrist as well as mild swelling at the fingertip area that suggested infection or an inflammatory lesion. Prior consultations with

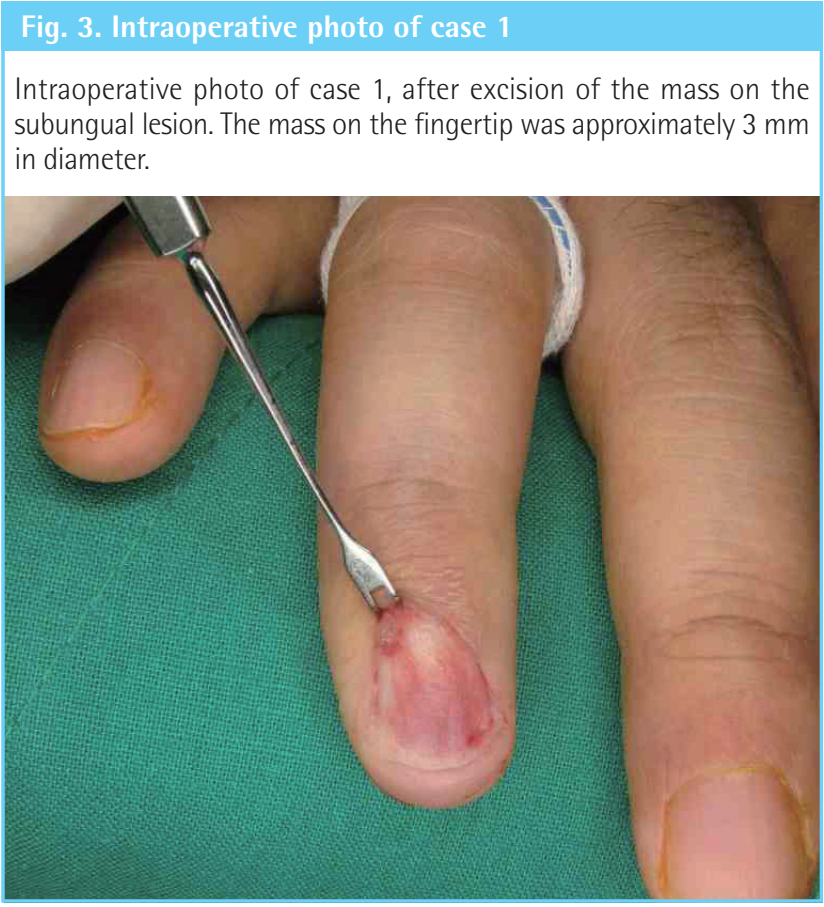

\section{Fig. 4. Preoperative photo of case 2}

This 78-year-old female patient suffered point pain, tenderness aggravated by cold irritation, and nail irregularity in the thumb. Treatment before with non-steroidal anti-inflammatory drugs, pain killers, and antibiotics did not alleviate the symptoms.

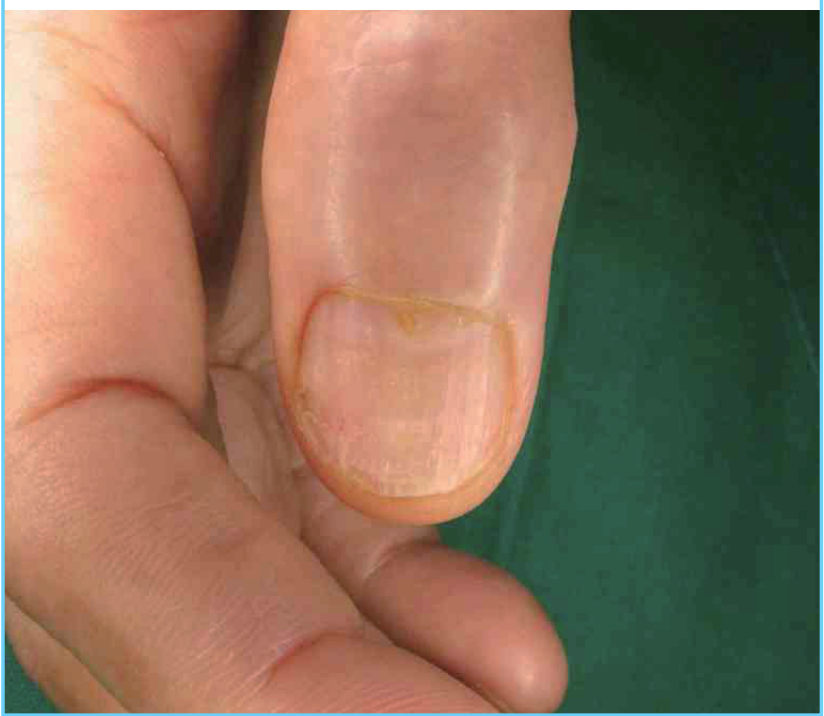


an orthopedic surgeon, pain clinic specialist, and also a plastic surgeon failed to establish a diagnosis. Pretreatment with nonsteroidal anti-inflammatory drugs, pain killers, and antibiotics did not alleviate the symptoms. At last, MR imaging was performed as a preoperative measure to eliminate the possibility of inflammatory or neurological disease. MR imaging revealed a hypo-intense well-defined nodule on the T1-weighted images, which appeared as hyper-intense lesions on the T2-weighted images of the thumb. Nodule enhancement also appeared with gadolinium (Fig. 5). No other abnormal lesions on the hand and forearm of this patient were observed. MR imaging suggested that the thumb nodular lesion was a glomus tumor. Finally,

\section{Fig. 5. Preoperative MRI of case 2}

Preoperative magnetic resonance imaging (MRI) of case 2 (T2weighted), which appeared as hyper-intense lesions (white arrow) on the thumb.

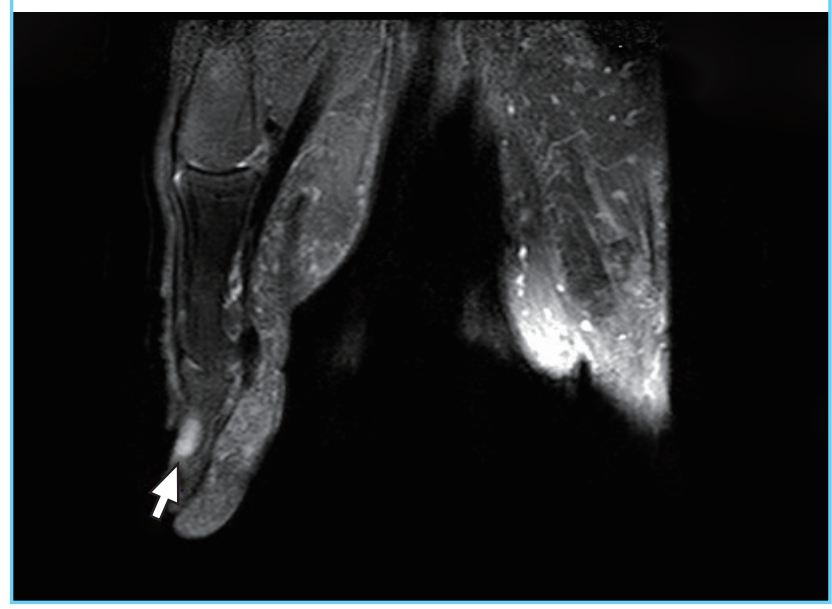

\section{Fig. 6. Intraoperative photo of case 2}

Intraoperative photo of case 2, after complete surgical excision.

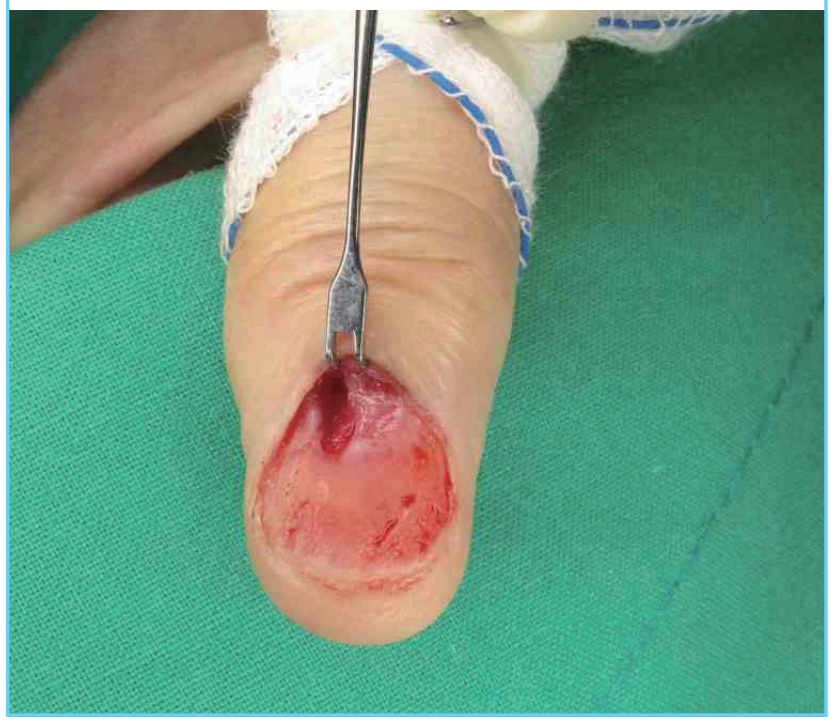

the lesion was surgically excised, and complete surgical excision followed by gross pathology revealed a well-defined red-blue nodule. Histopathology of the glomus tumor showed compact nests of monotonous polygonal cells with rounded nuclei and eosinophilic cytoplasm (Figs. 6-8). Following surgery, the patient was symptom-free without evidence of complications or recurrence.

\section{DISCUSSION}

The triad of localized pain, cold sensitivity, and point tenderness is characteristic of glomus tumors, although other hand tumors may mimic this clinical presentation [5]. Notably, early occult lesions of glomus tumor in the hand, particularly subungual

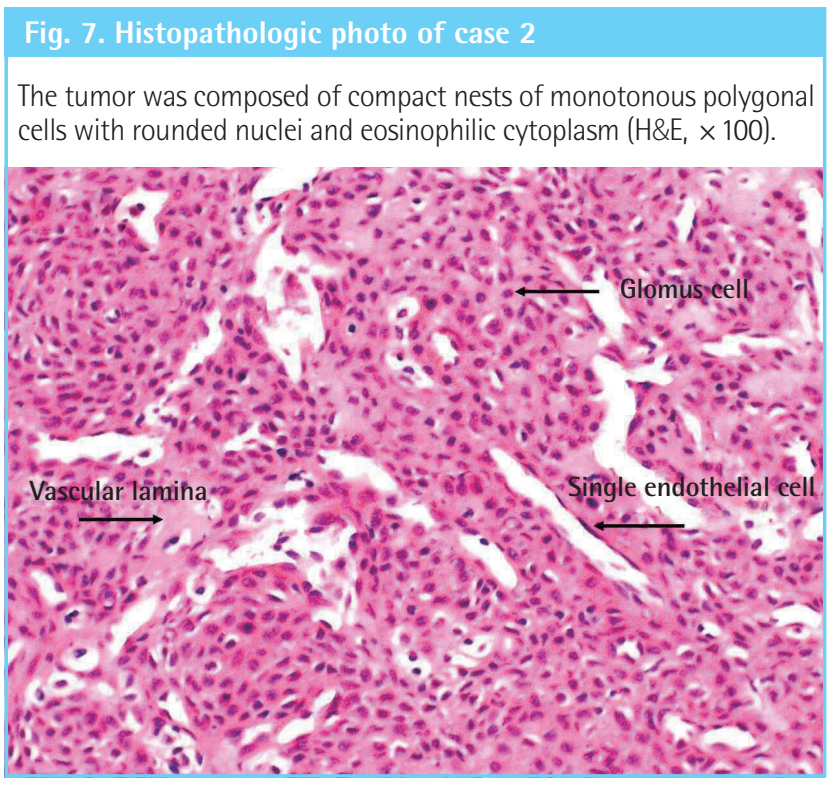

\section{Fig. 8. Histopathologic photo of case 2}

The tumor was composed of compact nests of monotonous polygonal cells with rounded nuclei and eosinophilic cytoplasm (H\&E, $\times 400)$.

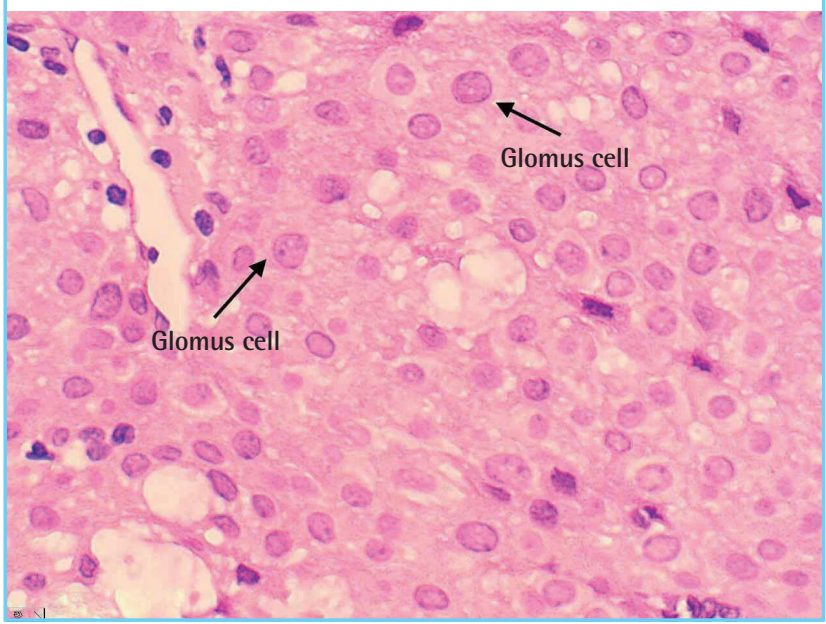


lesions, may not be identified by physical examination alone because the symptoms are difficult to detect [6]. Therefore, it is important to realize that sole dependence on physical examination alone for detecting glomus tumors may lead to misdiagnosis, delayed recognition, and inconsonant treatments, such as that for chronic nail infection or trigger finger.

As a preoperative imaging study for subungual glomus tumors, scintigraphy is a useful but nonspecific diagnostic tool. Moreover, ultrasonography is capable of demonstrating the size, site, and shape of the tumor, but is frequently influenced by the surgeon's experience. MR imaging, however, is an excellent examination tool for detecting the soft-tissue origin of a glomus tumor as small as $2 \mathrm{~mm}$. This method can also accurately define the location and limits of a tumor $[6,7]$.

Our cases demonstrate that MR imaging offers accurate diagnostic information in clinically undiagnosed or misdiagnosed patients. Preoperative MR imaging can accurately define the character and extent of glomus tumors, even though they are impalpable or invisible. However, it should be noted that Dahlin et al. [8] reported cases in which a glomus tumor was present despite a negative MR imaging result, indicating that MR imaging is not always accurate in displaying tumors.

As the current study asserts, preoperative MR imaging greatly assisted in diagnosing approximately $71.4 \%$ patients with unspecific or specific symptoms that prevented differential diagnosis between glomus tumor and other soft tissue lesions. Furthermore, all 21 cases examined in this study were clearly diagnosed by MR imaging, which revealed the localization of a hypointense lesion with a well-marginated soft tissue mass on T1weighted-images and that appeared as a brighter (higher signal intensity), hyper-intense lesion with a hypo-intense rim on T2weighted images.

Nevertheless, the possibility of a negative predictive value and the specificity of MR imaging findings must be taken into consideration. It was reported by Al-Qattan et al. [1] that the specificity and negative predictive value of MR imaging were low, indicating that a negative image does not rule out a glomus tumor in their study. However, in the present study, we found a positive predictive value for MR imaging that has greatly improved as of late, showing that MR can now detect lesions as small as $2 \mathrm{~mm}$.

Despite its controversy, MR imaging can clearly be helpful in diagnosing patients with obscure symptoms and signs. Therefore, a combination of clinical physical examinations and MR imaging should be performed for greater accuracy and early detection of subungual glomus tumors, and in order to correct misdiagnoses and treat tumors in the early stage for earlier relief of pain and accompanying symptoms.

In addition, we suggest that MR imaging can also be helpful in making differential diagnoses, including identifying a number of other lesions such as neuroma, melanoma, pigmented nevus, and hemangioma, as well as foreign bodies [9].

\section{REFERENCES}

1. Al-Qattan MM, Al-Namla A, Al-Thunayan A, et al. Magnetic resonance imaging in the diagnosis of glomus tumours of the hand.J Hand Surg Br 2005;30:535-40.

2. Drape JL, Idy-Peretti I, Goettmann S, et al. Subungual glomus tumors: evaluation with MR imaging. Radiology 1995; 195:507-15.

3. Sorene ED, Goodwin DR. Magnetic resonance imaging of a tiny glomus tumour of the fingertip: a case report. Scand J Plast Reconstr Surg Hand Surg 2001;35:429-31.

4. Theumann NH, Goettmann S, Le Viet D, et al. Recurrent glomus tumors of fingertips: MR imaging evaluation. Radiology 2002;223:143-51.

5. al-Qattan MM, Clarke HM. An isolated granular cell tumour of the thumb pulp clinically mimicking a glomus tumour. J Hand Surg Br 1994;19:420-1.

6. Koc O, Kivrak AS, Paksoy Y. Subungual glomus tumour: magnetic resonance imaging findings. Australas Radiol 2007;51 Spec No.:B107-9.

7. Espinosa-Gutierrez A, Izaguirre A, Baena-Ocampo L, et al. Images in Rheumatology. Glomus tumor. J Rheumatol 2009;36:1343-4.

8. Dahlin LB, Besjakov J, Veress B. A glomus tumour: classic signs without magnetic resonance imaging findings. Scand J Plast Reconstr Surg Hand Surg 2005;39:123-5.

9. Nazerani S, Motamedi MH, Keramati MR. Diagnosis and management of glomus tumors of the hand. Tech Hand Up Extrem Surg 2010;14:8-13. 